\title{
EVALUATION OF A MULTI-AXIAL, TEMPERATURE AND TIME DEPENDENT (MATT) FAILURE MODEL
}

\author{
D. E. Richardson, G. L. Anderson, and D. J. Macon \\ ATK Thiokol Propulsion, a Division of ATK Aerospace Company \\ Brigham City, UT 84302-0707
}

\begin{abstract}
To obtain a better understanding the response of the structural adhesives used in the Space Shuttle's

Reusable Solid Rocket Motor (RSRM) nozzle, an extensive effort has been conducted to characterize in detail the failure properties of these adhesives. This effort involved the development of a failure model that includes the effects of multi-axial loading, temperature, and time. An understanding of the effects of these parameters on the failure of the adhesive is crucial to the understanding and prediction of the safety of the RSRM nozzle. This paper documents the use of this newly developed multi-axial, temperature, and time (MATT) dependent failure model for modeling failure for the adhesives TIGA 321, EA913NA, and EA946. The development of the mathematical failure model using constant load rate normal and shear test data is presented. Verification of the accuracy of the failure model is shown through comparisons between predictions and measured creep and multi-axial failure data. The verification indicates that the failure model performs well for a wide range of conditions (loading, temperature, and time) for the three adhesives. The failure criterion is shown to be accurate through the glass transition for the adhesive EA946. Though this failure model has been developed and evaluated with adhesives, the concepts are applicable for other isotropic materials.
\end{abstract}

\section{Introduction}

Because of industrial safety and obsolescence issues, several adhesives (EA913NA and EA946) used in the Space Shuttle's RSRM nozzle (see Figure 1) are being replaced with an improved adhesive (TIGA 321). To ensure the safety and integrity of this TIGA 321 adhesive for use in a man-rated system, a thorough material characterization test program has been completed. This characterization was conducted on both the current adhesives and the replacement adhesive so that accurate comparisons could be made. Part of this study involved the evaluation of the effects of multi-axial loading, temperature, and time on failure. Traditionally, the effects of each of these conditions are accounted for separately. This paper documents the development and verification of a combined multiaxial, temperature, and time (MATT) dependent failure criterion for the three aforementioned adhesives. The criterion was developed with TIGA 321', EA913NA and EA946 in order to evaluate its applicability to multiple adhesive systems.

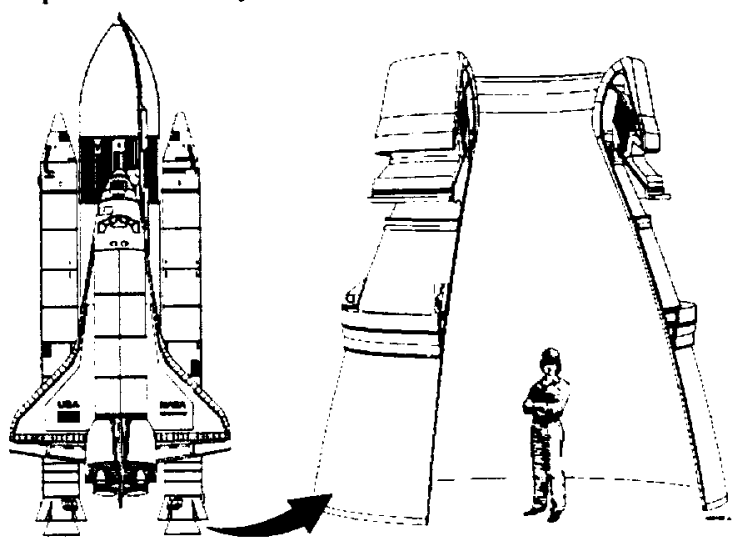

Figure 1: Space Shuttle RSRM Nozzle

\section{Multi-axial Temperature and Time (MATT)}

The Multi-Axial Temperature and Time (MATT) dependent failure criterion is of the following form:

$$
\mathrm{AP}^{2} \mathrm{~J}_{2}+\mathrm{BPI}_{1}=1
$$

Here, $J_{2}$ is the second deviatoric stress invariant, and $I_{1}$ is the first stress invariant. These invariants can be shown to be of the following form for an arbitrary stress state.

$$
\begin{gathered}
\mathrm{J}_{2}=\frac{1}{3}\left[\begin{array}{c}
\sigma_{11}^{2}+\sigma_{22}^{2}+\sigma_{33}^{2}- \\
\sigma_{11} \sigma_{22}-\sigma_{11} \sigma_{33}-\sigma_{22} \sigma_{33}+ \\
3 \sigma_{12}^{2}+3 \sigma_{13}^{2}+3 \sigma_{23}^{2}
\end{array}\right] \\
\mathrm{I}_{1}=\sigma_{11}+\sigma_{22}+\sigma_{33}
\end{gathered}
$$

$A$ and $B$ are parameters that define the shape of the failure envelope (which is ellipsoidal in nature). $P$ is the factor that is used to scale the size of the failure envelope to account for temperature and failure time effects. 